\title{
Beyond the Standard Model Searches Using a Free Electron Laser
}

\author{
A. Afanasev, ${ }^{\mathrm{a}}$ O.K. Baker, ${ }^{\mathrm{b}}$ K.B. Beard, ${ }^{\mathrm{c}}$ G. Biallas, ${ }^{\mathrm{d}}$ J. Boyce, ${ }^{\mathrm{d}}$ M. \\ Minarni, ${ }^{\mathrm{e}}$ R. Ramdon, ${ }^{\mathrm{a}}$ M. Shinn, ${ }^{\mathrm{d}}$ and P. Slocum ${ }^{\mathrm{b}}$ \\ ${ }^{a}$ Department of Physics, Hampton University, Hampton, VA 23668 \\ ${ }^{b}$ Department of Physics, Yale University, P.O. Box 208120, New Haven, CT 06520 \\ ${ }^{c}$ Muons, Inc., 552 N. Batavia Avenue, Batavia, IL 60510 \\ ${ }^{d}$ Free Electron Laser Division, Jefferson Laboratory, 12000 Jefferson Avenue, Newport News, VA 23606 \\ ${ }^{e}$ Department of Physics, Universitas Riau (UNRI), Pekanbaru, Riau 28293 Indonesia
}

\begin{abstract}
Much of the focus of Beyond the Standard Model physics searches is on the TeV scale, making use of hadron and lepton colliders. Additionally, however, there is the means to make these searches in different regions of parameter space using sub-electron volt photons from a Free Electron Laser, for example. We report on the experimental results of searches for opticalwavelength photons mixing with hypothetical hidden-sector paraphotons in the mass range between $10^{-5}$ and $10^{-2}$ electron volts for a mixing parameter greater than $10^{-7}$. We also report on the results of a sensitive search for scalar coupling of photons to light neutral bosons in the mass range of approximately 1.0 milli-electron volts and coupling strength greater than $10^{-6} \mathrm{GeV}^{-1}$. These were generation-regeneration experiments using the "light shining through a wall" technique in which regenerated photons are searched for downstream of an optical barrier that separates it from an upstream generation region. The present results indicate no evidence for photon-paraphoton mixing or for scalar couplings of bosons to photons for the range of parameters investigated.
\end{abstract}

Keywords: Dark Matter, Beyond the Standard Model, Hidden Sector

PACS: 11.30.Ly, 12.20.Fv, 12.60.Cn, 12.90.+b, 13.40.Hq

\section{OVERVIEW AND RESULTS}

If the Standard Model (SM) is part of a more fundamental theory which has some new mass scale, new dynamics and particles could appear and hence signal the new physics associated with it. Popular extensions of the SM motivated by string theory for example, predict a "hidden sector" of particles that interact with the "visible sector" SM fields only with feeble, gravitational-strength couplings [1-2]. This hidden sector can be probed using very high energy accelerators such as the Large Hadron Collider at the $\mathrm{TeV}$ scale, and also by laser experiments at the sub-electron volt (sub-eV) energy scale [3-13]. The importance of this study goes beyond even particle physics. A recent suggestion that paraphotons may give rise to a hidden cosmic microwave background (HCMB) [14] indicates that sub-eV particle physics may have direct bearing on cosmological studies. Additionally, several theories in particle physics as well as cosmology predict the existence of at least one scalar, that is, spin-zero, boson [9-19]. Many theories of physics beyond the SM (BSM) can accommodate scalars 
with very small masses and weak couplings to SM fields [15-19]. The experimental programs that explore the parameter space of weakly interacting, light, BSM vector and scalar bosons (ALPS at DESY, BMV at LULI, GammeV at FNAL, OSQAR at CERN, and LIPSS at JLab) by and large all use the "light shining through a wall” (LSW) technique of photon regeneration [20]. In the first case, laser photons kinetically mix with very weakly interacting vector paraphotons that the pass through an optical barrier (a "wall”) and then reconvert back into photons that are detected, as shown in Figure 1(a). In the second case, laser photons are sent through a strong magnetic field where some of them can convert into low-mass, weakly interacting bosons via the Primakoff effect, these bosons then pass through a wall that serves to block the incident laser light, and reconvert into photons in a second magnetic field in a similar manner, as shown in Figure 1(b).

\section{(a)}

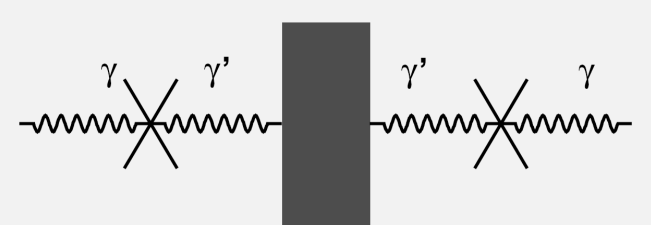

FIGURE 1. Photon regeneration using the "light shining through a wall" technique. (a) Photons $(\gamma)$ may convert into hidden-sector paraphotons $\left(\gamma^{\prime}\right)$ which proceed unimpeded through an optical barrier, reconvert back into photons downstream of the wall, and be detected in a properly executed experiment. The reconverted photons are expected to have the same properties as the original photons. (b) The incident light $(\gamma)$ couples to photons in the magnetic field (B) creating the hypothetical light neutral boson (A). Because it is weakly interacting, the boson passes through the optical barrier (the "wall") while no incident photons do so. Regenerated photons having the same characteristics as the original photons result from the second magnetic field region downstream of the wall as shown.

The Light Pseudoscalar and Scalar Search (LIPSS) collaboration took data that tests the $\gamma-\gamma^{\prime}$ mixing as well as Primakof conversion to light neutral bosons (LNBs) in a series of runs at the Jefferson Lab (JLab) Free Electron Laser (FEL) facility in Spring 2007. The details of these experimental runs are given in [21].

In the search for paraphotons via kinetic mixing with visible photons, the rate of regenerated photons, $r_{s}$, is given by

$$
r_{s}=r_{i} \cdot P_{\text {trans }} \cdot \frac{\Delta \Omega}{\Omega} \cdot \varepsilon
$$

where $r_{i}$ is the FEL (incident) photon rate, $\Delta \Omega / \Omega$ is the photon collection efficiency (solid angle for detection), $\varepsilon$ is the detector quantum efficiency, and [1]

$$
P_{\text {trans }}=16 \chi^{4}\left[\sin \left(\frac{\Delta k L_{1}}{2}\right) \sin \left(\frac{\Delta k L_{2}}{2}\right)\right]^{2}
$$

is the probability for photon regeneration from paraphotons that mix with incident photons in the generation region and propagate through the wall indicated in Figure 1(a). Here $\chi$ is the kinetic mixing parameter, $L_{1}\left(L_{2}\right)$ is the length of the generation (regeneration) region shown and the momentum difference between the photon and the hidden-sector paraphoton is defined as 


$$
\Delta k=\omega-\sqrt{\omega^{2}-m_{\gamma^{\prime}}{ }^{2}} \approx \frac{m_{\gamma^{\prime}}{ }^{2}}{2 \omega} \quad\left(\text { for } m_{\gamma^{\prime}}<<\omega\right)
$$

where $\omega$ is the laser photon energy $(1.33 \mathrm{eV})$ and $m_{\gamma}$ is the paraphoton mass. The Significance of the result is defined as $n_{s} / \sqrt{n_{b}}$ where $n_{s}$ is the number of signal events and $n_{b}$ is the number of background events. From this Significance, a 99\% Confidence Limit is calculated.
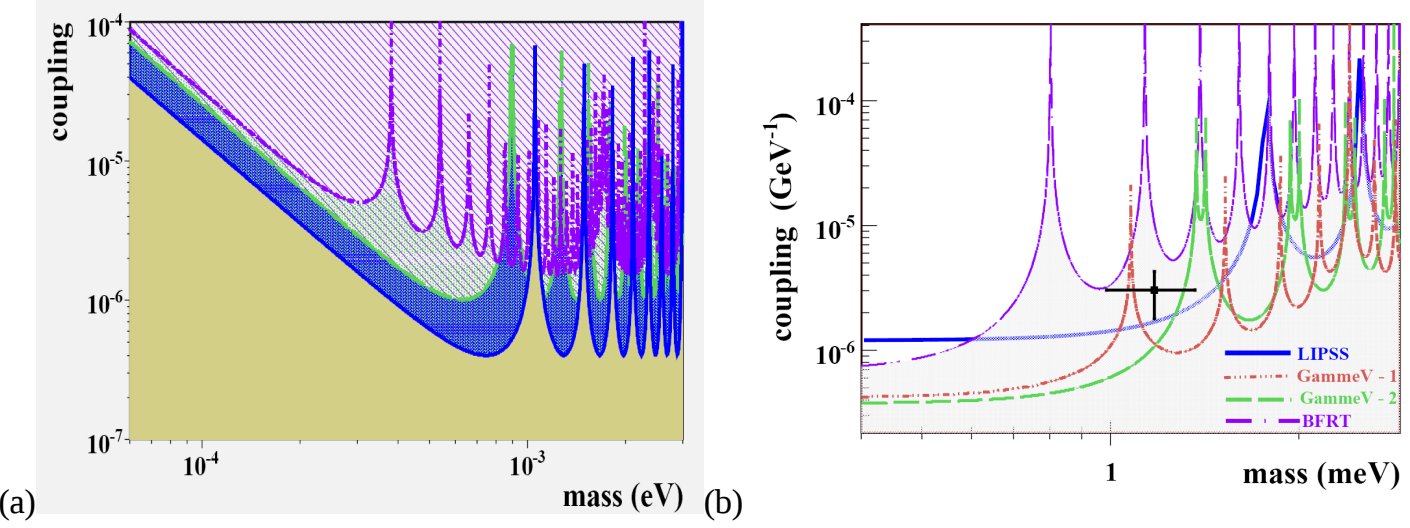

FIGURE 2. (a) A mixing parameter $\chi$ versus hidden sector paraphoton mass. Upper limits (95\% confidence) set by the recent "light shining through wall” experiments. The purple (upper) curve is from the BMV collaboration [23], the green (middle) curve is from the GammeV collaboration [22], and the blue curve is the new result from the LIPSS collaboration (99\%). The different structures for the curves correspond to the different photon energies and baselines used in the different experiments. (b) The new LIPSS limits on scalar coupling of photons to a hypothetical LNB versus the LNB mass in milli-electronvolts are shown in blue (full). The BFRT result [24] is shown (long dashed) in purple and the GammeV [22] results are shown in red and green(short dashed, and dot dashed). The region above the curves is ruled out in each case.

The results from this experiment can therefore be used to set the new limits on the mixing $\chi$ of visible photons to hypothetical hidden-sector paraphotons that are shown in Figure 2(a). The blue curve is the new LIPSS result, compared with those from the GammeV [22] and BMV [23] collaborations. The region above the curves is ruled out in each case.

In the presence of a magnetic field, the rate of regenerated photons, $\mathrm{r}_{\mathrm{s}}$, is given by

$$
r_{s}=r_{i} P_{\gamma \rightarrow L N B} P_{L N B \rightarrow \gamma} \frac{\Delta \Omega}{\Omega} \varepsilon
$$

where

$$
P_{\gamma \rightarrow L N B}=P_{L N B \rightarrow \gamma}=\frac{(g B)^{2}}{\frac{m^{4}}{4 \omega^{2}}} \sin ^{2}\left(\frac{m^{2} L}{4 \omega}\right) \approx \frac{1}{4}(g B L)^{2}
$$

is the probability of scalar boson generation from the incident photons for magnets not too long for a given wavelength of light; photon regeneration from these scalar particles is given by the identical expression as shown. Here $m(g)$ is the LNB mass 
(coupling strength to photons), and $B(L)$ is the magnetic field strength (length). The results from this run are used to set the new limits on the scalar coupling of photons to a hypothetical LNB shown in Figure 2(b). There are similar limits already set by the BMV [23], OSQAR [24], BFRT [25], and GammeV [22] collaborations for pseudoscalar and scalar couplings, but under slightly different LSW experimental conditions. The region above the curves is ruled out in each case. The data point is the region claimed (now disclaimed) by the PVLAS collaboration [26].

\section{ACKNOWLEDGMENTS}

The authors thank the technical staff of the Jefferson Lab Free Electron Laser Facility, especially F. Dylla, G. Neil, G. Williams, R. Walker, D. Douglas, S. Benson, K. Jordan, C. Hernandez-Garcia, and J. Gubeli, as well as M.C. Long of Hampton University for their excellent support of the experimental program. Funding from the Office of Naval Research Award K00141 is gratefully acknowledged.

\section{REFERENCES}

1. M. Ahlers et. al., Phys. Rev. D77 (2008) 095001.

2. J. Jaeckel and A. Ringwald, Phys. Lett. B659 (2008) 509.

3. B. Holdom, Phys. Lett. B166 (1986) 196.

4. R. Foot and X.G. He, Phys. Lett. B267 (1991) 509.

5. K.R. Dienes et. al., Nucl. Phys. B492 (1997) 104.

6. S.A. Abel and B.W. Schofield, Nucl. Phys. B685 (2004) 150.

7. L.B. Okun, Sov. Phys. JETP 56 (1982) 502.

8. V. V. Popov and O.V. Vasil'ev, Europhys. Lett. 15 (1991) 7.

9. M. Ahlers et. al., Phys. Rev. D75 (2007) 035011.

10. A. Ringwald, J. Phys. Conf. Ser. 39 (2006) 197.

11. J. Khoury and A. Weltman, Phys. Rev. Lett. 93 (2004) 171104; Phys. Rev. D69 (2004) 044026.

12. D.F. Mota and D.J Shaw, Phys. Rev. D75 (2007) 063501; Phys. Rev. Lett. 97 (2006) 151102.

13. G. Raffelt in W.-M Yao et. al., (Particle Data Group), J. Phys. G33 (2006) 1.

14. J. Jaeckel, J. Redondo, and A. Ringwald, Phys. Rev. Lett. 101 (2008) 131801; astro$\mathrm{ph} / 0804.4157 \mathrm{v} 1$ (2008).

15. Ph. Brax, C. van de Bruck, A.-C. Davis, J. Khoury, and A. Weltman, Phys. Rev. D70 (2004) 123518 ; Ph. Brax, C. van de Bruck, A.-C. Davis, J. Khoury, and A.M. Green, Phys. Lett. B633 (2006) 441.

16. K. Choi, Phys. Rev. D62 (2000) 043509.

17. I. Waga and J. Frieman, Phys. Rev. D62 (2000) 043521; J. Frieman et. al., Phys. Rev. Lett. 75 (1995) 2077; J.A. Frieman, C.T. Hill, and R. Watkins, Phys. Rev. D46 (1992) 1226.

18. R.D. Pecci and H.R. Quinn Phys. Rev. Lett. 38 (1977) 1440; Phys. Rev. D16 (1977) 1791; S. Weinberg, Phys. Rev. Lett. 40 (1978) 223; F. Wilczek, Phys. Rev. Lett. 40 (1978) 279.

19. D. Maity, S. Roy, and S. SenGupta, Phys. Rev. D77 (2008) 015010.

20. K. V. Bibber et. al., Phys. Rev. Lett. 59 (1987) 759.

21. A. Afanasev et.al. Phys. Phys. Lett. 101 (2008) 120401; Phys. Lett. B679 (2009) 317.

22. A. Chou et. al., Phys. Rev. Lett. 100 (2008) 080402.

23. M. Fouche et. al., Phys. Rev D78 (2008) 032013; C. Robilliard et. al., Phys. Rev. Lett. 99 (2007) 190403.

24. R. Ballou et. al., CERN Report, CERN-SPEC-2007-039 (2007).

25. R. Cameron et. al., Phys. Rev. D47 (1993) 3707.

26. E. Zavattini et. al., Phys. Rev. Lett. 96 (2006) 110406.; http://axion-wimp.desy.de/index eng.html (2007). 